\title{
Transtorno mental e sofrimento psíquico: representações sociais de profissionais da
}

\section{Atenção Básica à Saúde}

\author{
Mental disorder and psychic suffering: social representations of primary health care professionals \\ Trastorno mental y sufrimiento psíquico: representaciones sociales de profesionales de la salud \\ básica
}

Recebido: 24/02/2021 | Revisado: 02/03/2021 | Aceito: 07/03/2021 | Publicado: 14/03/2021

\author{
Pamela dos Santos Farinhuk \\ ORCID: https://orcid.org/0000-0003-1472-2920 \\ Faculdades Pequeno Príncipe, Brasil \\ E-mail: pamelafarinhuk@gmail.com \\ Luciana Elisabete Savaris \\ ORCID: https://orcid.org/0000-0002-7408-1187 \\ Faculdades Pequeno Príncipe, Brasil \\ E-mail: lucianaesavaris@gmail.com \\ Renato Soleiman Franco \\ ORCID: https://orcid.org/0000-0003-1176-480X \\ Pontifícia Universidade Católica do Paraná, Brasil \\ E-mail: paum@uol.com.br
}

\begin{abstract}
Resumo
A Atenção Básica à Saúde é a porta de entrada preferencial ao Sistema Único de Saúde e um dos pontos de atenção à saúde mental da população. O objetivo deste estudo foi compreender as representações sociais do sofrimento psíquico e dos transtornos mentais para profissionais que atuam em equipes de referência de Unidades Básicas de Saúde com Estratégia de Saúde da Família e identificá-las frente às influências na tomada de decisão quanto à elaboração do plano terapêutico. Trata-se de uma pesquisa qualitativa, descritivo-exploratória com delineamento transversal, baseada na teoria das representações sociais desenvolvida por Serge Moscovici. Os dados foram coletados entre abril e junho de 2020, em um distrito sanitário de saúde de uma capital do sul do país, por meio de entrevista semiestruturada e questionário sociodemográfico. Participaram do estudo 17 trabalhadores entre eles médicos/as, enfermeiros/as e auxiliares/técnicos/as de enfermagem. Os resultados apontam que existe uma mudança de paradigma em andamento, embora os profissionais encontrem limitações tanto na formação quanto institucionais para exercer o cuidado integral em saúde mental. Conclui-se que os profissionais da Atenção Básica à Saúde diferenciam os conceitos de transtorno mental e sofrimento psíquico, entretanto isso não impacta significativamente na tomada de decisão sobre o plano terapêutico, fato que se correlaciona a estrutura assistencial vigente, a qual demanda dos profissionais intervenções rápidas e resolutivas, que implica na escolha preferencial por uma terapêutica medicamentosa e encaminhamentos a serviços especializados.
\end{abstract}

Palavras-chave: Saúde mental; Atenção básica à saúde; Sofrimento psíquico; Transtorno mental; Representação social.

\begin{abstract}
Primary Health Care is the preferred gateway to the Unified Health System and one of the attention points to the population's mental health. The aim of this study was to understand the social representations of psychic suffering and mental disorders for professionals who work in reference teams of Basic Health Units with a Family Health Strategy and to identify them in the face of influences on decision-making regarding the elaboration of the therapeutic treatment plan. It is a qualitative, descriptive-exploratory research with a cross-sectional design, based on the theory of social representations developed by Serge Moscovici. Data were collected between April and June 2020, in a health district of a southern capital of the country, through semi-structured interviews and a socio-demographic questionnaire. 17 workers participated of the study, including doctors, nurses and nursing assistants / technicians. The results indicate that there is a paradigm shift in progress, although professionals find limitations both in training and in institutions to exercise comprehensive mental health care. It is concluded that the professionals of Primary Health Care differentiate the concepts of mental disorder and psychic suffering, however this does not significantly impact the decision-making about the therapeutic treatment plan, a fact that correlates to the current assistance structure, which demands on professional fast and resolutive interventions, which implies the preferential choice for drug therapy and referral to specialized services.
\end{abstract}

Keywords: Mental health; Primary Health care; Psychic suffering; Mental disorder; Social representation. 


\begin{abstract}
Resumen
La Atención Primaria de Salud es la puerta de entrada de preferencia al Sistema Único de Salud y uno de los puntos de atención a la salud mental de la población. El objetivo de este estudio fue comprender las representaciones sociales del sufrimiento psíquico y de los trastornos mentales para profesionales que trabajan en equipos de referencia de Unidades Básicas de Salud con una Estrategia de Salud de la Familia e identificar si estas representaciones influyen en las decisiones referentes a elaboración del plan terapéutico. Se trata de una investigación cualitativa, descriptivaexploratoria con un diseño transversal, basada en la teoría de las representaciones sociales desarrollada por Serge Moscovici. Los datos han sido recogidos entre abril y junio de 2020, en un distrito de salud de una capital del sur del país, mediante entrevistas semiestructuradas y un cuestionario sociodemográfico. En el estudio participaron 17 trabajadores, entre médicos, enfermeras y auxiliares / técnicos de enfermería. Los resultados indican que existe un cambio de paradigma en progreso, aunque los profesionales descubren limitaciones tanto en la formación como en las instituciones para ejercer una atención integral en salud mental. Se concluye que los profesionales de Atención Primaria de Salud diferencian los conceptos de trastorno mental y sufrimiento psíquico, sin embargo esto no impacta significativamente en las decisiones sobre el plan terapéutico, hecho que se correlaciona con la estructura asistencial vigente, la cual demanda intervenciones profesionales rápidas y resolutivas, que implica en la selección por una terapéutica famacológica y la condución a servicios especializados.
\end{abstract}

Palabras clave: Salud mental; Primeros auxilios; Sufrimiento psíquico; Trastorno mental; Representación social.

\title{
1. Introdução
}

A construção do modelo de atenção à saúde mental no Sistema Único de Saúde (SUS) está entrelaçada à história da Saúde Coletiva, que tem suas origens intimamente ligadas à luta pela democracia e ao movimento da Reforma Sanitária Brasileira (Osmo \& Schriber, 2015).

O movimento da Reforma Psiquiátrica Brasileira (RPB), iniciou a partir da década de 1970, motivado pelas deflagrações de violação de direitos e práticas iatrogênicas que ocorriam nos hospitais psiquiátricos. Influenciada pelo modelo italiano de Franco Basaglia, surge a proposta de descentralização do cuidado (Amarante \& Nunes, 2018; Hirdes, 2009) que culminou na aprovação da Lei n. 10.216/01 (Brasil, 2001).

Correlato à construção do modelo de atenção à saúde mental, o SUS desenvolve no ano de 1994 o Programa de Saúde da Família (PSF), que passa a ser adotado como estratégia de caráter mais abrangente no ano de 2006 (Pinto \& Giovanella, 2018), reafirmando a Atenção Básica à Saúde (ABS) como principal porta de entrada e ordenadora do cuidado (Brasil, 2000).

Em 2011, a Portaria n. 3.088 (Brasil, 2011), que implementa a Rede de Atenção Psicossocial (RAPS), destaca as Unidades Básicas de Saúde (UBS) como dispositivo de atenção à saúde mental (Rocha, 2016), que desempenha um importante papel tanto na busca de diminuir o estigma e preconceito, como no processo de desinstitucionalização de pacientes com transtornos mentais graves e crônicos, viabilizando o cuidado em território (Brasil, 2013; Lima, Severo, Andrade, Soares \& Silva, 2013; Arce, Sousa \& Lima, 2011).

Estudos como os de Ribeiro, Gianini, Goldbaum e Cesar (2018) evidenciam que as queixas relacionadas aos transtornos mentais e/ou sofrimento psíquico são frequentes na ABS, sendo que as mais prevalentes estariam relacionadas ao sofrimento psíquico, presentes em até 50,3\% da população brasileira. Segundo Aragão et al. (2018), em pesquisa realizada em UBS com Estratégia de Saúde da Família (ESF), o atendimento a queixas de saúde mental chega a 19,48\% do total da demanda, desses $43 \%$ sofrimento psíquico e $34,7 \%$ transtornos mentais graves, impactando diretamente na qualidade de vida que é avaliada pior em usuários com essas queixas, do que na população em geral.

Tendo em vista esta realidade, torna-se iminente discutir como tais demandas de saúde mental são acolhidas na ABS pelas diferentes categorias profissionais que compõem as equipes de referência (Silva et al., 2019), sendo o acolhimento entendido como diretriz constitutiva do modo de produzir saúde e ferramenta de intervenção, garantia de acesso e resolutividade nos serviços (Pizzinato, Jesus \& Pagnussat, 2012).

Para subsidiar essa discussão torna-se necessário algum consenso em relação ao campo da saúde mental, que circunscreva tanto os transtornos mentais como o sofrimento psíquico. A saúde mental é uma área extensa, complexa e 
transversal do conhecimento, campo polissêmico e plural, produto de múltiplas interações entre fatores biológicos, psíquicos e sociais; refere-se à percepção que as pessoas têm sobre suas vidas, da busca pelo bem estar e possibilidade de enfrentamento do cotidiano (Alves \& Rodrigues, 2010).

Ainda que se tenha alcançado avanços no cuidado à saúde mental, são muitos os desafios que permeiam as equipes da ABS acerca destas demandas, dentre estes, Arce, Sousa e Lima (2011) apontam a falta de apropriação de ferramentas de intervenção que tendem a gerar uma escassez de ofertas terapêuticas reprimindo a demanda existente. Outro fator a ser considerado é a falta de autonomia das equipes, refletindo diretamente em processos de trabalho que mantem os moldes dos antigos padrões assistenciais (Arce, Sousa \& Lima, 2011; Silveira \& Vieira, 2009).

Sabidamente estes profissionais são influenciados tanto por um paradigma biomédico - organicista, prescritivo e intervencionista - quanto pela transição de um modelo hospitalocêntrico para um modelo psicossocial no que tange o cuidado em saúde mental (Gaino, Souza, Cirineu \& Tulimosky, 2018; Fertonani, Pires, Biff \& Scherer, 2015; Rocha \& David, 2015). Este estudo objetiva compreender as representações sociais do sofrimento psíquico e dos transtornos mentais de profissionais da equipe de referência que atuam na ABS e identificá-las frente as influências na tomada de decisão quanto à elaboração do plano terapêutico.

\section{Metodologia}

Trata-se de uma pesquisa de abordagem qualitativa, exploratório-descritiva com delineamento transversal, realizada entre os meses de abril a junho de 2020. Segundo Gerhardt e Silveira (2009) a pesquisa qualitativa tem como principais características descrever, compreender e explicar os fenômenos observando as diferenças entre o mundo social e o natural. Já o caráter exploratório-descritivo torna o problema mais explícito e visível, possibilitando a construção de hipóteses.

O aporte teórico metodológico adotado para alcançar os objetivos propostos foi o da teoria das Representações Sociais desenvolvida por Serge Moscovici (1961), que estimula a compreensão do conhecimento produzido. O estudo de uma representação social pressupõe "investigar o que pensam, por que pensam e como pensam os indivíduos" (Bertoni \& Galinkin, 2017, p.106). A compreensão de um conceito é obtida através das percepções subjetivas e representações sociais construídas acerca dele (Sousa; Maciel \& Medeiros, 2018; Moscovici, 2015), por fim, as representações sociais podem ser consideradas fenômenos sociais dinâmicos e heterogêneos, que são construídos, preservados e alterados através da comunicação e da linguagem, essa teoria pressupõem a transformação do conhecimento (Marková, 2017).

Para efeitos desta pesquisa, serão considerados transtornos mentais, aqueles que afetam o indivíduo e comprometem sua funcionalidade, etiologicamente apresentam indicações genéticas e químicas e encontram-se definidos no Manual de Diagnóstico e Estatístico de Transtornos Mentais (DSM) e na Classificação Internacional de Doenças (CID) (Andrade et al., 2016). Quando identificados na ABS demandam intervenções especializadas, tais como: avaliação e suporte do Núcleo de Apoio à Saúde da Família (NASF) com categorias da saúde mental, acompanhamento médico, prescrição medicamentosa e mesmo encaminhamentos/articulação para serviços de maior complexidade como os Centros de Atenção Psicossocial (CAPS) e Hospitais (Brasil, 2013).

Quanto ao sofrimento psíquico, considera-se aquele que se relaciona aos desafios cotidianos. Pode se mostrar a partir de sintomas tais como: esquecimento, dificuldade na concentração e na tomada de decisões, insônia, cansaço e ansiedade, além de queixas somáticas (Andrade et al., 2016). Sabe-se que essa condição gera muitos prejuízos, inclusive o risco aumentado de transtornos mentais (Prata et al., 2017). Nesses casos as ofertas precisam extrapolar o paradigma biomédico, ou seja, demandam de profissionais da saúde a utilização de tecnologias leves como escuta, acolhimento, articulações intra e intersetoriais que viabilizem Projetos Terapêuticos Singulares (PTS) e que envolvem abordagem sobre os determinantes sociais da saúde (DSS) e busquem ampliar o repertório do indivíduo e desenvolver sua autonomia (Santos; Mishima \& Merhy, 2018). 
Esta pesquisa foi realizada em três UBS com ESF em um dos 10 Distritos Sanitários (DS) de Saúde do município de Curitiba, localizado na região sul. A área de abrangência desse DS tem uma população de 143.433 habitantes. Esse DS é composto por 12 UBS, sendo que 11 delas possuem ESF, uma Unidade de Pronto Atendimento, um Centro de Atenção Psicossocial (modelo territorial, atendendo usuários de álcool e drogas, assim como pessoas com demandas de transtornos mentais) e um hospital municipal (Marques, 2018). As unidades selecionadas para coleta possuem o Índice de Vulnerabilidade das Áreas de Abrangência das Unidades Municipais de Saúde (IVAB) registrado como médio, que de acordo com o Decreto $\mathrm{n}^{\circ}$ 638, de 21 de junho e 2018, da Secretaria Municipal da Saúde de Curitiba, refere-se ao calculado da média aritmética entre os índices de quatro dimensões: adequação do domicílio, perfil e composição familiar, acesso ao trabalho e renda, condições de escolaridade (Souza, 2020).

As UBS foram escolhidas por conveniência, visto que a pesquisadora se encontrava inserida na qualidade de Residente Multiprofissional nas mesmas. Participaram dessa pesquisa 17 trabalhadores/as nas seguintes funções e número de participantes: médicos/as (cinco), enfermeiros/as (três) e auxiliares/técnicos/as de Enfermagem (nove).

O convite para integrar a pesquisa foi realizado pessoalmente pela pesquisadora, tendo como critério de inclusão: atuar por no mínimo um ano em ESF, estar alocado em uma das três unidades selecionadas para o estudo, ter mais que 18 anos, aceitar participar da pesquisa e assinar o Termo de Consentimento Livre e Esclarecido (TCLE). Foram excluídos/as os/as participantes que atuavam em ESF a menos de um ano, que não estavam lotados/as nas UBS selecionadas para pesquisa, que possuíam vínculo de residência com a UBS, que estavam afastados/as durante o período de coleta por férias ou outro tipo de licença, que não aceitaram participar da pesquisa ou que durante a coleta desejaram interromper por qualquer motivo.

A entrevista foi realizada após a explicação dos direitos de cada participante, leitura conjunta, esclarecimento de eventuais dúvidas relativas ao TCLE e assinatura do mesmo, sendo que uma das vias foi fornecida a/ao participante e a outra permaneceu em posse da pesquisadora.

A coleta das informações foi realizada a partir de um questionário sociodemográfico e entrevista semiestruturada. O roteiro continha as seguintes questões disparadoras: "defina transtorno mental"; "defina sofrimento psíquico" e "descreva como o transtorno mental e sofrimento psíquico se apresentam na sua prática diária na UBS".

As entrevistas foram realizadas individualmente, em sala reservada. Do total, 15 foram gravadas mediante autorização dos/as participantes e posteriormente transcritas na íntegra. Dois dos/as participantes não autorizaram gravação, por isso essas entrevistas foram registradas por escrito no momento de sua realização. $\mathrm{O}$ encerramento da amostra deu-se por saturação de dados, quando novas entrevistas não apresentavam dados diferentes dos já obtidos.

Optou-se pela análise das informações através da Análise de Conteúdo, proposta pela professora Laurence Bardin, que apresenta três fases elementares: pré-análise, exploração do material e tratamento dos resultados - a inferência e a interpretação (CÂMARA, 2013).

Esse projeto foi aprovado pelo Comitê de Ética em Pesquisa da instituição proponente, com parecer consubstanciado favorável sob o n. 3.850.640 e Certificado de Apresentação para Apreciação Ética sob o n. 27133319.8.0000.0101.

\section{Resultados e Discussão}

Após análise do conteúdo das entrevistas emergiram três categorias principais que norteiam a discussão: Modelo assistencial: Entre o ideal e o Possível; Formação em saúde mental das diferentes categorias que integram a equipe de Referência da ABS e Sofrimento Psíquico: objetividade versus subjetividade.

\section{Categoria 1 - Modelo assistencial: Entre o ideal e o Possível}

Nesta primeira categoria é possível observar as representações sociais dos transtornos mentais e do sofrimento psíquico 
dos profissionais e a rotina do cuidado a saúde mental na ABS. As narrativas apontam para uma representação social dos transtornos mentais associada a diagnósticos clínicos, intervenções medicalizantes e direcionamento do cuidado para pontos especializados.

"Transtorno mental é uma alteração que a pessoa tem num momento da vida e ela necessita de um acompanhamento, de um tratamento, seja ele terapêutico, através de acompanhamento psicológico ou através de medicamentoso" (P 4)

"Transtorno mental é tudo quanto é percepção que foge do comum né, tipo assim, a pessoa tem um transtorno de ansiedade, como ela é classificada, cada um tem uma classificação né? Uns são ansiosos, uns são psicóticos, outros são esquizofrênicos. Então assim, a gente percebe que cada um tem um nível né, um grau, um nível pra cada" (P 8)

"Transtorno mental eu entendo que é um conjunto de doenças que são da mente né, da parte neurológica assim" (P 13)

"[O tratamento...] É primeiro o medicamentoso, que é horrivel isso, porque vai aumentando as doses né, começa com uma fluoxetina, uma sertralina, uma coisa assim, dali a pouco vai indo né e nunca mais sai [...]” (P 15)

"[...] aqui mais o tratamento é com remédios né. Aqui pra gente né, no caso de vocês tratamento e saber do sofrimento, essas coisas são mais no psiquiatra mesmo, no consultório mesmo, pra você atender uma pessoa bem legal assim, só tendo uma conversa bem diferenciada com ela" (P8)

Enquanto a representação social do sofrimento psíquico, identifica uma relação causal entre o sofrimento e aspectos externos, situações de vida e nível individual de suportabilidade das situações adversas:

"tem vários tipos, por exemplo quando criança é abusada, ameaçada a não contar, agressão, mulher também quando apanha do marido" (P10)

"Sofrimento psíquico pra mim é alguém ficar é, manipulando você e você não ter uma liberdade de poder escapar daquele sofrimento" (P12)

"O sofrimento psíquico, ele vem através de situações que as pessoas não conseguem lidar, a partir do momento que elas não conseguem lidar com algumas questões, se dá o sofrimento psíquico. Que pode vir desde coisas muito simples, como por exemplo, dificuldade de lidar com as doenças, doença na família, questões vividas, drogadição, essas coisas que causam sofrimento psíquico" [...] (P15)

Os achados relacionados a intervenções medicalizantes podem ser encontrados em outros estudos similares, como o de Fegadolli, Varela e Carlini (2019) que indica altas taxas de uso de benzodiazepínicos na ABS. Embora a medicação seja uma das estratégias possíveis no cuidado a pessoas com transtornos mentais, chama a atenção quando a prescrição de psicofármacos é priorizada no tratamento do sofrimento psíquico, inclusive em casos de quadros sem a existência de um registro diagnóstico, como apontam Borges, Hegadoren e Miasso (2015). Essa realidade parece ser usual (Borges, Miasso, Vedana, Telles Filho \& Hegadoren, 2015) e segundo Bezerra, Jorge, Gondim, Lima e Vasconcelos (2014) cada vez mais frequente a escolha pelo tratamento medicamentoso para aspectos sociais, econômicos e existenciais, indicando um baixo uso de tecnologias leves na ABS.

A escolha preferencial pelo tratamento medicamentoso e direcionamento a serviços especializados, contraria a lógica psicossocial que busca uma atenção centrada no usuário, na compreensão de seu sofrimento, de suas condições psicológicas, econômicas e sociais e dos seus desafios e possibilidades terapêuticas. O modelo psicossocial prioriza ações no próprio território, pautadas no vínculo entre usuários e profissionais, acompanhamento longitudinal e apoio matricial - arranjo organizacional que concebe suporte técnico em áreas específicas às equipes responsáveis pelo desenvolvimento de ações básicas em saúde (Pizzinato et al., 2012).

A identificação de uma rotina que diferencia transtornos mentais de sofrimentos psíquicos, mas que demostra 
intervenções prioritariamente baseadas no modelo biomédico, trata de uma realidade bastante complexa e multifatorial. Gerbaldo, Arruda, Horta e Garnelo (2018) justificam a partir da ausência de protocolos para o atendimento a demandas de transtornos mentais na ABS e o pouco investimento em qualificação das equipes multiprofissionais. Lima e Guimarães (2019), para além do já citado, referem o preconceito existente acerca do transtorno mental. Esses autores indicam como razões para manutenção do modelo de atenção centrado na doença, o fato do trabalho em ESF ser direcionado a programas específicos, derivados de políticas focalizadoras que não se comunicam entre si; e a formulação dos processos de trabalho, que geralmente impõem restrições de tempo, dificultando o uso de tecnologias leves, como acolhimento e a escuta (Lima \& Guimarães, 2019). Pizzinato et al. (2012) também citam a falta de diretrizes do Ministério da Saúde, a falta de preparo técnico do profissional, as precárias condições de trabalho e a falta de investimento de gestores.

Somando-se às questões apontadas na literatura, os participantes desta pesquisa destacam ainda a sobrecarga da ABS como barreira para o uso de tecnologias leves:

"[...] a agenda da gente é, tem muitos pacientes né. E a gente tem vários programas, tem as gestantes, tem a criança, o idoso, o hipertenso, o diabético, e tudo é prioridade pra secretaria e a saúde mental também é, é uma fatia ali do pão também, e claro que é importante também" (P7).

Além da percepção de despreparo dos profissionais ao atendimento de queixas relacionadas a saúde mental conforme retratado pelo participante:

“[...] a grande maioria não tá preparada pra atender a pessoa que tá com transtorno e muito menos com quem tá em sofrimento, porque falta empatia com esse tipo de atendimento e falta sensibilidade pra você discernir e saber também abordar a pessoa" (P9).

São várias as contradições entre o ideal e o possível na rotina das UBS:

“Eu vejo que a psicologia ajuda bastante, mas é bem restrito. O NASF ajuda, mas eu digo mais em relação a falta de vagas assim sabe [...] por exemplo, o paciente que precisa de uma terapia mesmo com o psicólogo, a gente não tem tantas vagas disponiveis né?" (P5)

"Até identifico, até consigo, o problema é você ter tempo pra manejar isso ai né, não tem, porque a demanda é grande e você não consegue fazer como você gostaria." (P2)

"Tem muita função, muita demanda e a gente não consegue ter um atendimento que você fique no mínimo uns 20 minutos de atendimento dentro do consultório pra tentar entender a pessoa." (P9)

Trajano (2017) relaciona a situação de sobrecarga e consequente cansaço dos profissionais com as quebras no trabalho em rede, a desistência no cuidado, a intensificação no encaminhamento, desencadeado pela sensação de "não dar conta". O que contribui para a perpetuação do modelo de atenção centrado na doença, sendo executado em um ciclo permanente, independentemente da percepção e reconhecimento dos profissionais acerca de intervenções não farmacológicas e da atenção centrada na pessoa, pois fica evidenciado que esse conhecimento não tem espaço nos limites institucionais. Conforme exposto na fala de P15:

"O dia a dia dificulta, a agenda, o tempo de consulta, que eu acho que é muito pouco tempo pra você resolver assim de conversar, escutar, a escuta né que precisa ser feita primeiro. É assim, só tem o tal do QP né, a queixa principal, o que que você veio fazer aqui hoje?" 


\section{Categoria 2- Formação em saúde mental das diferentes categorias que integram a equipe de Referência da ABS}

Dentre os achados, a formação em saúde mental das diferentes categorias estudadas, médicos, enfermeiros, técnicos e auxiliares de enfermagem, emergiu como questão a ser problematizada quando se preconiza no modelo psicossocial que a UBS seja a porta de entrada preferencial para as queixas relacionadas a saúde mental e que profissionais da equipe de referência sejam os primeiros a receberem essas demandas (Fagundes; Campos \& Fortes, 2019; Brasil, 2012).

Entre os participantes oito possuem formação em nível superior (enfermeiros e médicos) e nove em nível médio (auxiliares/técnicos de enfermagem), essa diferença de escolaridade entre os profissionais é um dado importante, pois o acesso a conteúdo de saúde mental é distinto entre cursos técnicos e de graduação. Espera-se que auxiliares e técnicos de enfermagem tenham disciplinas específicas como Enfermagem Psiquiátrica e Saúde Mental. Entretanto Zerbertto e Pereira (2009) apontam em seus estudos que os cursos abordam os conhecimentos referentes a sintomatologia psiquiátrica e diagnóstico para identificação da pessoa com transtorno mental, sem levar em conta tecnologias leves de cuidado, não foi possível identificar muitos estudos que abarquem a formação de saúde mental para cursos técnicos.

Quanto a graduação em enfermagem, Vargas, Maciel, Bittencourt, Lenate e Pereira (2018) indicam que as grades curriculares vêm passando por mudanças de paradigma, assim como, o próprio modelo assistencial em saúde mental, no entanto, ainda predominam disciplinas com enfoque biomédico, legado do antigo paradigma flexneriano no ensino dos cursos de graduação da área da saúde, que compreendem o biologicismo/organicismo como determinante do processo saúde-doença tanto em nível individual quanto coletivo (Rodrigues, Kempfer, Lenz \& Oliveira, 2017).

O curso de graduação em medicina é tradicionalmente pautado em um modelo de aprendizagem biomédico, entretanto a partir da criação do SUS, com necessidade de recursos humanos para atuação nessa área, essa estruturação passou a ser reformulada (Carneiro \& Porto, 2014). Nas Diretrizes Curriculares do curso de graduação em Medicina Cavalcanti, Gomes e Azevedo (2020) informam que no ano de 2014 a saúde mental passa a ser área obrigatória do internato, com o objetivo de ampliar o saber e a prática médica para um olhar do sofrimento humano como fenômeno que não pode ser reduzido a somente a uma classificação diagnóstica.

Os profissionais apontam a insuficiência da abordagem do tema saúde mental em suas formações o que segundo Sousa, Maciel, Medeiros e Vieira (2016) e Schneider, Pereira e Ferraz (2018) impacta no manejo das queixas na rotina, tendendo a gerar um afastamento e a delegar a intervenção a um profissional que supostamente está mais qualificado para o atendimento. Silveira, Costa e Jorge (2018) alertam sobre os riscos de se desenvolver uma atuação superficial e pouco resolutiva quando o campo de percepção clínica está reduzido, ou seja, com foco na queixa e intervenções pontuais, desconsiderando a complexidade do indivíduo.

“a gente pede apoio pra psicologia, porque tem coisa que a nossa formação não dá conta, eu não tenho um conhecimento pra ajudar. A gente tenta dar apoio, oferecer ajuda, tenta encaminhar, mas tem coisa que tem um limite da formação da gente, que a gente não consegue às vezes entender, é difícil, tem o limite que a gente tem outra formação né. A gente vai mais pra parte biológica né, a parte química né, e às vezes tem que ir pra essa parte subjetiva aí." (P7)

"[...] quando você faz, por exemplo enfermagem, você tem várias ramificações da graduação né. Então ou você se especializa em saúde mental e tem o domínio do assunto, porque ser generalista acaba dificultando um pouco, porque você às vezes não sabe achar o ponto certo de abordar a pessoa e de identificar." (P9)

A formação específica em saúde mental, embora apresente uma diferença em relação às demais formações em saúde, também reflete ainda em sua práxis referenciais teóricos e metodológicos herdados do modelo biomédico, em oposição à prática pautada no modelo de assistência psicossocial (Sousa et al., 2018).

Sousa et al. (2016) em pesquisa realizada com estudantes universitários dos cursos de Psicologia, Enfermagem e 
Medicina, sobre as representações sociais destes acerca da RPB e de doentes mentais, demonstraram que embora o paradigma psicossocial venha ganhando espaço desde o início do processo da RPB, ainda não está plenamente estabelecido, e nem o paradigma biomédico completamente superado, persistem ainda representações de medo e exclusão em relação ao doente mental (Sousa et al., 2016).

Identificar e problematizar as representações sociais contemporâneas no campo da Saúde Mental é um caminho na busca de superar o modelo de atenção centrado no transtorno. Mas, além disso, são fundamentais as constantes reavaliações das práticas vigentes no cuidado ao usuário com transtorno mental, a instrumentalização de profissionais de saúde de todas as categorias e promoção de políticas públicas que além de assegurar direitos, também pautem a qualidade no cuidado (Braga \& Farinha, 2018).

\section{Categoria 3 - Sofrimento psíquico: objetividade versus subjetividade}

Por fim, a terceira categoria de análise discute o sofrimento psíquico atravessado pela objetividade e subjetividade dos indivíduos. No contexto da ABS a identificação do sofrimento psíquico pode ser um desafio, exigindo das equipes de saúde o uso de tecnologias leves, como a escuta, o acolhimento, a empatia, o vínculo, que perpassam pelo processo de entendimento subjetivo do profissional (Oliveira, Suto \& Silva, 2016; Brasil, 2013). Cabe considerar que queixas de sofrimento psíquico frequentemente não são apresentadas de maneira direta por usuários/as, e sim, a partir de queixas orgânicas tais como: inapetência, desconfortos gástricos, alterações no sono, entre outras. A falta de relatos diretos ocorre em vista do preconceito ainda vigente acerca de questões psíquicas, que são interpretadas como fraqueza, manipulação ou falha de caráter em nossa sociedade e não como uma dimensão do sujeito que interfere diretamente no processo saúde-doença (Silva et al., 2019).

Portanto, a subjetividade do profissional durante o contato inicial com o usuário torna-se uma ferramenta importante para identificação do sofrimento apresentado, conforme relatado pelos participantes:

"[...] sofrimento psíquico ele é obviamente subjetivo, então assim, claro que tem uma subjetividade que é uma subjetividade do profissional que atende né, como ele percebe aquele sofrimento, e obviamente que sofrimento que dialogue com a gente, a gente percebe de forma mais clara às vezes né do que sofrimento que não dialogue com a nossa experiência de vida e o sofrimento que o paciente relata como sofrimento." (P17)

"Geralmente eles vem misturados né. O transtorno mental ele parece ter uma, é, parece critérios definidos assim, sabe. Já o sofrimento psíquico ele vai mais da análise da pessoa, isso durante a consulta às vezes quando vem os dois juntos daí você consegue diferenciar." (P5)

Destaca-se nos achados a percepção dos/as profissionais de que o vínculo entre profissionais e usuário/a se torna essencial tanto para identificação do sofrimento psíquico, como para seu manejo. Oliveira et al. (2016) reiteram que o vínculo é fundamental ao trabalho da equipe de referência ESF, considerando que os profissionais acompanham longitudinalmente seus usuários. Como explicitam:

“[...] porque a gente tem um vínculo com a família né, principalmente aqueles pacientes que estão sempre aqui. Eles se abrem com a gente, eles têm assim essa liberdade a maioria, de se abrir com a gente, quando a gente dá espaço pra eles, né" (P14).

"O manejo depende de cada paciente, tem alguns que dão brecha pra gente perguntar, outros chegam direto pedindo ajuda. Outros chegam fechados e percebemos que não é normal, não é o jeito do paciente. Eles chegam na gente e falam tudo que estão sentindo, o vínculo faz diferença" (P1).

"O manejo aqui na atenção básica é a gente com a gente mesmo. A gente é linha de frente. Tem apoios de psiquiatria, de psicologia, mas é mais a gente com a gente mesmo. [...] como a gente tem mais contato eles acabam se apegando a 
gente, e isso facilita muito o manejo" (P5).

Contudo, apesar das potencialidades mencionadas, dificuldades práticas/objetivas prejudicam a identificação e manejo do sofrimento psíquico na $\mathrm{ABS}$, conforme exposto pelos participantes da pesquisa:

"[sobre dificuldades do dia a dia] é o tempo né, 40 pessoas ou mais pra gente conversar (com cada uma) se precisar de uma palavra né” (P3).

“E ás vezes a agenda da gente não permite ficar muito tempo com um paciente desse que demanda muito tempo, né [...] A agenda do enfermeiro é uma agenda infinita, que não tem fim. Se chegar 50 o enfermeiro tem que atender 50 $[\ldots] "$ ".. (P7).

"O nosso tempo é mais corrido, a gente não tem tempo pra dar uma atenção valorizada, humana para o paciente né, porque é muitos pra atender, então o nosso é mais quantidade, né. Bem isso que eu sinto assim” (P8).

“[...] na prática diária não dá tempo de a gente conversar o suficiente, né [...] é porque ninguém vai abrir tua vida, os sofrimentos, os traumas é, em 15 minutos sabe [...]” (P13).

A escuta tem papel fundamental no cuidado humanizado em saúde mental, é uma tecnologia que auxilia no entendimento empático da subjetividade do indivíduo (Santos, 2019), contudo, a relação escuta e tempo de atendimento demonstra ser uma das grandes barreiras no SUS.

O tempo médio da consulta médica e de enfermagem é estipulado conforme indicadores e pactuação local, ou seja, cada secretaria tem liberdade para adaptar o fluxo de trabalho conforme a realidade local, estipulando metas, visando operacionalização do trabalho em ESF (Brasil, 2012). Esse planejamento tem reflexos diretos na prática diária e tomada de decisão quanto ao plano terapêutico, conforme descrito por P15:

"é difícil isso, primeiro por causa do tempo mesmo, da cobrança de números, números e números, isso dificulta. [...] É números, a secretaria quer números, e quando fala em trabalho com sofrimento psíquico não pode ter números, é tempo, e vai na medicalização mesmo, porque parece que resolve, mas não resolve, é um paliativo [...]”.

Lima, Vieira e Silveira (2015) em estudo realizado com enfermeiros/as atuantes em serviços de saúde mental, afirmam que a escuta terapêutica é uma ferramenta pouco utilizada pelos/as profissionais, atribuem isso a constante preocupação com o tempo de atendimento.

Por fim, este estudo destaca o risco trazido por Braga e Farinha (2018) de reduzir o indivíduo ao sintoma, em detrimento de um olhar sobre a pessoa e o contexto em que está inserida ao não se efetivar uma apropriação aprofundada da queixa do usuário/a, assim patologizando experiências humanas. Esse foco na sintomatologia abre espaço para a medicalização do sofrimento, ou medicalização social, em que problemas sociais e/ou situações de vida diversas que geram sofrimento, passam a ser tratados como doenças (Tesser, 2019; Van Dijk, Faber, Tanke, Jeurissen \& Westert, 2016).

\section{Considerações Finais}

Conclui-se que as representações sociais dos profissionais das equipes de referência da ABS referente aos transtornos mentais está associada a diagnósticos clínicos enquanto o sofrimento psíquico se vincula a situações de vida como violências e outras vulnerabilidades. Isso permite inferir que profissionais da ABS os diferenciam, contudo, essa distinção não parece repercutir na tomada de decisão quanto à elaboração do plano terapêutico que tende a priorizar em ambos os casos a escolha por tratamentos medicalizantes e encaminhamentos a serviços especializados. Estas escolhas terapêuticas parecem estar fundamentadas nas barreiras encontradas pelos profissionais para utilização de tecnologia leves de cuidado, em destaque as 
limitações de tempo de atendimento e pressão por resultados em metas. Além da falta de apropriação/formação, obstáculos que refletem na perpetuação do modelo de cuidado centrado na queixa.

As contribuições deste estudo foram as de compreender as representações sociais de profissionais da ABS e identificar as contradições entre o modelo psicossocial vigente e as possibilidades de sua efetivação na prática das UBS. Considera-se que para além da formação em saúde mental, que deve qualificar as diferentes categorias profissionais que atuam nas equipes de referência, torna-se necessário problematizar o processo de trabalho das equipes e suas condições de trabalho, visando romper barreiras estruturais que inviabilizam a efetivação do modelo psicossocial.

O estudo apresentou limitações, sendo que o momento da coleta de dados pode ser considerado como o mais desafiador dessa pesquisa em função do cenário de pandemia (Sars-CoV-2) vivido. Foi necessária a adoção de protocolos de segurança para realização das entrevistas, além de encontrar trabalhadores angustiados/as e inseguros pelas alterações constantes nos processos de trabalho e o próprio temor pessoal de adoecer.

Esta pesquisa despertou o interesse em ampliar a escuta de profissionais em outros territórios do município, para identificar similaridades e dissimilitudes, surgindo o interesse em dar continuidade ao estudo e tendo uma visão geral da capital paranaense.

Por fim, considera-se que os objetivos de estudo foram alcançados, o que permite refletir que o cuidado efetivo e de qualidade em saúde mental no SUS, é um trabalho contínuo, que envolve formação, modelo assistencial e subjetividade. Parafraseando um dos participantes desta pesquisa: "não existem soluções simples para questões complexas" (P17).

\section{Agradecimentos}

Agradecemos ao Programa de Residência Multiprofissional de Saúde da Família da Secretaria Municipal da Saúde de Curitiba.

\section{Referências}

Alves, A. A. M., \& Rodrigues, N. F. (2010). Determinantes sociais e econômicos da saúde mental. Revista Portuguesa de Saúde Pública, 28(2), 127-131. http://www.scielo.mec.pt/scielo.php?script=sci_arttext\&pid=S0870-90252010000200003

Amarante, P., \& Nunes, M. O. (2018). A reforma psiquiátrica no SUS e a luta por uma sociedade sem manicômios. Ciência \& Saúde Coletiva, 23(6), 20672074. https://www.scielosp.org/pdf/csc/2018.v23n6/2067-2074/pt

Andrade, A. S., Tiraboschi, G. A., Antunes, N. A., Viana, P. V. B. A., Zanoto, P. A., \& Curilla, R. T. (2016). Vivências acadêmicas e sofrimento psíquico de estudantes de psicologia. Psicologia: Ciência e Profissão, 36(4), 831-846. https://www.scielo.br/scielo.php?script=sci_arttext\&pid=S1414$98932016000400831 \& \operatorname{lng}=\mathrm{pt} \& \operatorname{tlng}=\mathrm{pt}$

Aragão, E. I. S., Campos, M. R., Portugal, F. B., Gonçalves, D. A., Mari, J. J., \& Fortes, S. L. C. L. (2018). Padrões de apoio social na atenção primária à saúde: diferenças entre ter doenças físicas ou transtornos mentais. Ciência \& Saúde Coletiva, 23(7), 2339-2350. http://www.scielo.br/scielo.php?pid=S1413$81232018000702339 \&$ script=sci_abstract\&tlng=pt

Arce, V. A. R., Sousa, M. F., \& Lima, M. G. (2011) A práxis da saúde mental no âmbito da estratégia de saúde da família: contribuições para a construção de um cuidado integrado. Physis - Revista de Saúde Coletiva, 21(2), 541-560. http://www.scielo.br/scielo.php?script=sci_arttext\&pid=S0103-73312011000200011

Bertoni, L. M., \& Galinkin, A. L. (2017). Teoria e métodos em representações sociais. In: Mororó, L. P., Couto, M. E. S., \& Assis, R. A. M. orgs. Notas teóricometodológicas de pesquisas em educação: concepções e trajetórias [online]. (pp. 101-122). http://books.scielo.org/id/yjxdq/pdf/mororo-9788574554938-05.pdf

Bezerra, I. C., Jorge, M. S. B., Gondim, A. P. S., Lima, L. L., \& Vasconcelos, M. G. F. (2014). "Fui lá no posto e o doutor me mandou foi pra cá": processo de medicamentalização e (des)caminhos para o cuidado em saúde mental na Atenção Primária. Interface. 18(48), 61-74. https://www.scielo.br/pdf/icse/v18n48/1807-5762-icse-18-48-0061.pdf

Borges, T. L., Miasso, A. I., Vedana, K. G. G., Telles Filho, P. C. P., \& Hegadoren, K. M. (2015). Prevalência do uso de psic otrópicos e fatores associados na atenção primária à saúde. Acta Paulista de Enfermagem, 28(4), 344-349. https://www.scielo.br/pdf/ape/v28n4/1982-0194-ape-28-04-0344.pdf

Borges, T. L., Hegadoren, K. M., \& Miasso, A. I. (2015). Transtornos mentais comuns e uso de psicofármacos em mulheres atendidas em unidades básicas de saúde em um centro urbano brasileiro. Revista Panamericana de Salud Publica, 38(3), 195-201. https://scielosp.org/pdf/rpsp/2015.v38n3/195-201/pt

Braga, T. B. M., \& Farinha, M. G. (2018). Sistema único de saúde e a reforma psiquiátrica: desafios e perspectivas. Revista da Abordagem Gestáltica, 24(3), 366-378. http://pepsic.bvsalud.org/scielo.php?script=sci_arttext\&pid=S1809-68672018000300009 
Câmara, R. H. (2013). Análise de conteúdo: da teoria à prática em pesquisas sociais aplicadas às organizações. Gerais: Revista Interinstitucional de Psicologia, 6(2), 179-191. http://pepsic.bvsalud.org/pdf/gerais/v6n2/v6n2a03.pdf

Carneiro, L. A., \& Porto, C. C. (2014). Saúde mental nos cursos de graduação: interface com as diretrizes curriculares nacionais e com a reforma psiquiátrica. Cadernos Brasileiros de Saúde Mental, 6(14), 150-167. p/cbsm/article/view/68544\#: :text=As\%20Diretrizes\%20Curriculares\%20Nacionais\%20para,de\%20servi\%C3\%A7os\%20abertos\%20e\%20comunit\%C3\%A1 rios.

Cavalcanti, M. T., Gomes, M. K., \& Azevedo, L. M. S. (2020). Internato de Saúde Mental para alunos de Medicina: qual o melhor cenário de formação?. Interface: comunicação, saúde, educação, 24, 1-13. https://www.scielo.br/scielo.php?script=sci_arttext\&pid=S1414-32832020000100500\&tlng=pt

Fagundes, G. S., Campos, M. R., \& Fortes, S. L. C. L. (2019). Matriciamento em saúde mental - análise do cuidado às pessoas em sofrimento psíquico na atenção básica. Ciência e Saúde Coletiva, periódico na internet. http://www.cienciaesaudecoletiva.com.br/artigos/matriciamento-em-saude-mental-analise-docuidado-as-pessoas-em-sofrimento-psiquico-na-atencao-basica/17333?id=17333\&id=17333\& $\mathrm{Id}=17333$

Fegadolli, C., Varella, N. M. D., \& Carlini, E. L. A. (2019). Uso e abuso de benzodiazepínicos na atenção primária à saúde: práticas profissionais no Brasil e em Cuba. Cadernos de Saúde Pública, 35(6), 1-11. https://www.scielo.br/scielo.php?script=sci_arttext\&pid=S0102-311X2019000705007\&lang=pt

Fertonani, H. P., Pires, D. E. P., Biff, D., \& Scherer, M. D. A. (2015). Modelo assistencial em saúde: conceitos e desafios para a atenção básica brasileira. Ciência \& Saúde Coletiva, 20(6), 1869-1878. https://www.scielo.br/scielo.php?script=sci_arttext\&pid=S1413-81232015000601869

Gaino, L. V., Souza, J., Cirineu, C. T., \& Tulimosky, T. D. (2018). O conceito de saúde mental para profissionais de saúde: um estudo transversal e qualitativo. SMAD -Revista Eletrônica Saúde Mental Álcool e Drogas, 14(2), 108-116. http://pepsic.bvsalud.org/pdf/smad/v14n2/07.pdf

Gerbaldo, T. B., Arruda, A. T., Horta, B. L., \& Garnelo, L. (2018). Avaliação da organização do cuidado em saúde mental na atenção básica à saúde do Brasil. Trabalho, Educação e Saúde, 16(3), 1079-1094. https://www.scielo.br/pdf/tes/v16n3/1678-1007-tes-1981-7746-sol00150.pdf

Gerhardt, T. E., \& Silveira, D. T. (2009). Método de pesquisa. Editora da UFRGS. http://www.ufrgs.br/cursopgdr/downloadsSerie/derad005.pdf

Hirdes, A. (2009). A reforma psiquiátrica no Brasil: uma (re) visão. Ciência \& Saúde Coletiva, 14(1), 297-305. https://www.scielo.br/scielo.php?pid=s1413$81232009000100036 \&$ script=sci_abstract\&tlng=pt

Lei n. 10.216/01, de 06 de abril de 2001. Dispõe sobre a proteção e os direitos das pessoas portadoras de transtornos mentais e redireciona o modelo assistencial em saúde mental. Presidência da República. http://www.planalto.gov.br/ccivil_03/leis/leis_2001/110216.htm

Lima, A. I. O., Severo, A. K., Andrade, N. L., Soares, G. P., \& Silva, L. M. (2013). O desafio da construção do cuidado integral em saúde mental no âmbito da atenção primária. Temas em Psicologia, 21(1), 71-82. http://pepsic.bvsalud.org/scielo.php?script=sci_arttext\&pid=S1413-389X2013000100005

Lima, D. K. R. R., \& Guimarães, J. (2019). Articulação da rede de atenção psicossocial e continuidade do cuidado em território: problematizando possíveis relações. Physis: Revista de Saúde Coletiva, 29(3), 1-20. https://www.scielo.br/pdf/physis/v29n3/0103-7331-physis-29-03-e290310.pdf

Lima, D. W. C., Vieira, A. N., \& Silveira, L. C. (2015). A escuta terapêutica no cuidado clínico de enfermagem em saúde mental. Revista Texto Contexto Enfermagem, 24(1), 154-160. https://www.scielo.br/pdf/tce/v24n1/pt_0104-0707-tce-24-01-00154.pdf

Marková, I. A. (2017). A fabricação da teoria de representações sociais. Cadernos de Pesquisa, 47(163), 358-375. https://www.scielo.br/pdf/cp/v47n163/19805314-cp-47-163-00358.pdf

Marques, A. P. M. (2018). Violências contra os adolescentes em um distrito sanitário: as potencialidades e fragilidades para efetivar a linha de Cuidado na rede de proteção. [Dissertação de Mestrado, Universidade Federal do https://acervodigital.ufpr.br/bitstream/handle/1884/58558/R\%20-\%20D\%20-\%20ANA\%20PAULA\%20MACHADO\%20MARQUES.pdf?sequence=1\&isAllo wed=y

Ministério da Saúde. (2000). Programa saúde da família. Secretaria de Atenção à Saúde, Departamento de Atenção Básica. http://bvsms.saude.gov.br/bvs/publicacoes/implantacao_unidade_saude_familia_cab1.pdf

Ministério da Saúde. (2012). Política Nacional de Atenção Básica. Série E. Legislação em Saúde. http://189.28.128.100/dab/docs/publicacoes/geral/pnab.pdf

Ministério da Saúde. (2012). Saúde Mais Perto de Você - Acesso e Qualidade programa nacional de melhoria do acesso e da qualidade da atenção básica (PMAQ). Departamento de Atenção Básica. http://189.28.128.100/dab/docs/publicacoes/geral/manual_instrutivo_pmaq_site.pdf

Ministério da Saúde. (2013). Acolhimento à demanda espontânea. Departamento de Atenção Básica. http://bvsms.saude.gov.br/bvs/publicacoes/acolhimento_demanda_espontanea_cab28v1.pdf

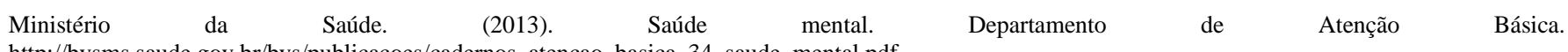
http://bvsms.saude.gov.br/bvs/publicacoes/cadernos_atencao_basica_34_saude_mental.pdf

Moscovici, S. (2015). Representações sociais: investigações em psicologia social. Vozes.

Oliveira, J. S. B., Suto, C. S. S., \& Silva, R. S. (2016). Tecnologias leves como práticas de enfermagem na atenção básica. Revista Saúde.com, 12(3), 613-621. http://periodicos2.uesb.br/index.php/rsc/article/view/425

Osmo, A., \& Schraiber, L. B. (2015). O campo da saúde coletiva no Brasil: definições e debates em sua constituição. Saúde e Sociedade, 24(1), 205-218. https://www.scielo.br/pdf/sausoc/v24s1/0104-1290-sausoc-24-s1-00205.pdf

Pinto, L. F., \& Giovanella, L. (2018). Do programa à estratégia saúde da família: expansão do acesso e redução das internações por condições sensíveis à atenção básica (ICSAB). Ciência \& Saúde Coletiva, 23(6), 1903-1913. https://www.scielosp.org/pdf/csc/2018.v23n6/1903-1914/pt 
Pizzinato, A., Jesus, L. O., \& Pagnussat, E. (2012). Avaliação de demandas e abordagens de atenção em saúde mental em unidade com estratégia de saúde da família. Revista $\quad$ Psicologia $\quad e \quad$ Saúde, $\quad 1-9$. https://repositorio.pucrs.br/dspace/bitstream/10923/8990/2/Avaliacao_de_demandas_e_abordagens_de_atencao_em_saude_mental_em_unidade_com_estrateg ia_de_saude_da_familia.pdf

Portaria $\mathrm{n}^{\mathrm{0}}$ 3.088, de 23 de dezembro de 2011. Institui a Rede de Atenção Psicossocial para pessoas com sofrimento ou transtorno mental e com necessidades decorrentes do uso de crack, álcool e outras drogas, no âmbito do Sistema único de Saúde (SUS). Ministério da Saúde. http://bvsms.saude.gov.br/bvs/saudelegis/gm/2011/prt3088_23_12_2011_rep.html

Prata, N. I. S. S., Groisman, D., Martins, D. A., Rabello, E. T., Mota, F. S., Jorge, M. A., Nogueira, M. L., Calicchio, R. R., \& Vasconcelos, R. V. (2017). Saúde mental e atenção básica: território, violência e o desafio das abordagens psicossociais. Trabalho, Educação e Saúde, 15(1), 33-53. https://www.scielo.br/scielo.php?pid=S1981-77462017000100033\&script=sci_abstract\&tlng=pt

Ribeiro, M. K. P., Gianini, R. J., Goldbaum, M., \& Cesar, C. L. G. (2018). Equidade na cobertura dos gastos com saúde pelo Sistema Único de Saúde de pessoas com indicativos de transtornos mentais comuns no município de São Paulo. Revista Brasileira de Epidemiologia, 21(1), 1-13. https://www.scielo.br/scielo.php?pid=S1415-790X2018000100410\&script=sci_abstract\&tlng=pt

Rodrigues, J., Kempfer, S. S., Lenz, J. R., \& Oliveira, S. N. (2017). Influência das reformas curriculares no ensino de saúde mental em enfermagem: 1969 a 2014. Revista Gaúcha de Enfermagem, 38(3), 1-9. https://www.scielo.br/scielo.php?script=sci_arttext\&pid=S1983-14472017000300407

Rocha, P. R., \& David, H. M. S. L. (2015). Determinação ou determinantes? Uma discussão com base na teoria da produção social da saúde. Revista da Escola de Enfermagem da USP, 49(1), 129-135. http://www.scielo.br/pdf/reeusp/v49n1/pt_0080-6234-reeusp-49-01-0129.pdf

Rocha, R. F. (2016). Representações sociais da saúde mental. Revista Eletrônica Científica Inovação e Tecnologia, 2(14), 95-113. https://periodicos.utfpr.edu.br/recit/article/view/4298

Santos, A. B. (2019). Escuta qualificada como ferramenta de humanização do cuidado em saúde mental na Atenção Básica. APS em Revista, 1(2), 170-179. https://apsemrevista.org/aps/article/view/23

Santos, D. S., Mishima, S. M., \& Merhy, E. E. (2018). Processo de trabalho na estratégia de saúde da família: potencialidades da subjetividade do cuidado para reconfiguração do modelo de atenção. Ciência e Saúde coletiva, 23(3), 861-870. http://www.scielo.br/pdf/csc/v23n3/1413-8123-csc-23-03-0861.pdf

Schneider, L. R., Pereira, R. P. G., \& Ferraz, L. (2018). A prática baseada em evidência no contexto da Atenção Primária à Saúde. Saúde debate, 42(118), 594605. https://www.scielo.br/scielo.php?script=sci_arttext\&pid=S0103-11042018000300594

Silva, P. M. C., Costa, N. F., Barros, D. R. R. E., Silva Junior, J. A., Silva, J. R. L., \& Brito, T. S. (2019). Saúde mental na atenção básica: possibilidades e fragilidades do acolhimento. Revista Cuidarte, 10(1), 1-12. https://revistacuidarte.udes.edu.co/index.php/cuidarte/article/view/617

Silveira, C. B., Costa, L. S. P., \& Jorge, M. S. B. (2018). Redes de Atenção à Saúde como produtoras de cuidado em saúde mental: Uma análise reflexiva. Revista Portuguesa de Enfermagem de Saúde Mental, (19), 61-70. http://www.scielo.mec.pt/pdf/rpesm/n19/n19a08.pdf

Silveira, D. P., \& Vieira, A. L. S. (2009). Saúde mental e atenção básica em saúde: análise de uma experiência no nível local. Ciência \& Saúde Coletiva, 14(1), 139-148. https://www.scielosp.org/pdf/csc/2009.v14n1/139-148/pt

Sousa, P. F., Maciel, S. C., Medeiros, K. T., \& Vieira, G. L. S. (2016). Atitudes e Representações em Saúde Mental: Um Estudo com Universitários. Psico USF, 21(3), 527-538. https://www.scielo.br/scielo.php?script=sci_arttext\&pid=S1413-82712016000300527\&lang =pt

Sousa, P. F., Maciel, S. C., \& Medeiros, K. T. (2018). Paradigma biomédico x psicossocial: onde são ancoradas as representações sociais acerca do sofrimento psíquico? Temas em Psicologia, 26(2), 883-895. https://www.scielo.br/pdf/tpsy/v26n2/2358-1883-tpsy-26-02-0883.pdf

Souza, M. N. (2020). O índice de vulnerabilidade das unidades municipais de saúde como estratégia de enfrentamento da COVID-19 na periferia de Curitiba. Observatório das Metrópoles. https://www.observatoriodasmetropoles.net.br/o-indice-de-vulnerabilidade-das-unidades-municipais-de-saude-como-estrategiade-enfrentamento-da-covid-19-na-periferia-de-curitiba/\#: :text=De\%20acordo\%20com\%20o\%20Decretoadequa\%

C3\%A7\%C3\%A3o\%20do\%20domic\%C3\%ADlio\%2C\%20perfil \%20e

Tesser, C. D. (2019). Cuidado clínico e sobremedicalização na atenção primária à saúde. Trabalho Educação e Saúde, 17(2), 1-27. https://www.scielo.br/pdf/tes/v17n2/1678-1007-tes-17-02-e0020537.pdf

Trajano, M. P. (2017). O cuidado em saúde mental: limites e possibilidades da rede de atenção psicossocial. [Dissertação de Mestrado, Universidade Federal de Santa Catarina]. https://repositorio.ufsc.br/handle/123456789/188992

Van Dijk, W., Faber, M. J., Tanke, M. A. C., Jeurissen, P. P. T., \& Westert, G. P. (2016). Medicalisation and overdiagnosis: what society does to medicine. International Journal of Health Policy and Management, 5(11), 619-622. https://www.ncbi.nlm.nih.gov/pmc/articles/PMC5088721/

Vargas, D., Maciel, M. E. D., Bittencourt, M. N. Lenate, J. S., \& Pereira, C. F. (2018). O ensino de enfermagem psiquiátrica e saúde mental no brasil: análise curricular da graduação. Texto Contexto Enfermagem, 27(2), 1-9. https://www.scielo.br/pdf/tce/v27n2/0104-0707-tce-27-02-e2610016.pdf

Zerbetto, S. R. \& Pereira, M. A. O. (2009). O Ensino de saúde mental e psiquiatria nos cursos técnicos de enfermagem. Revista Mineira de Enfermagem, 13(3), 358-364. https://cdn.publisher.gn1.link/reme.org.br/pdf/v13n3a07.pdf 